\title{
Subarachnoid haemorrhage and myths about saccular aneurysms
}

\author{
R O Weller
}

\section{Introduction}

The incidence of subarachnoid haemorrhage is about 10:100000 per year in Western Europe and USA and comprises some $10 \%$ of all cases of cerebrovascular disease. ${ }^{1}$ Forty five per cent of patients die within the first 30 days following subarachnoid haemorrhage; nearly two thirds die as a result of the initial haemorrhage and most within the first two days. ${ }^{2}$ Despite vigorous therapeutic efforts, the mortality rate from subarachnoid haemorrhage remains high. A greater understanding of the basic mechanisms and pathology of subarachnoid haemorrhage may lead to more effective prevention and therapeutic strategies.

This leader reviews the causes and results of subarachnoid haemorrhage and examines how myths surrounding subarachnoid haemorrhage and saccular aneurysms have been dispelled by the introduction of new techniques and more reliable clinical and epidemiological data.

\section{Outcome of subarachnoid haemorrhage}

Subarachnoid haemorrhage occurs mainly between the ages of 40 and 60 years and is more common in women than men by a proportion of $2-3: 1 .^{3}$ Characteristically, there is a sudden onset of severe headache, frequently concentrated in the occipital region; patients may or may not lose consciousness. Diagnosis is made by detecting blood in the subarachnoid space by computed tomography (CT) or by the presence of fresh or altered blood in the cerebrospinal fluid (CSF). The mortality rate in the first two days is $28 \%$, mostly from the initial bleed; $45 \%$ of patients are dead by 30 days, either from the initial bleed or from rebleeding. ${ }^{2}$

Most deaths from subarachnoid haemorrhage occur rapidly and the mortality rate is proportional to the amount of blood released. Those who have a haemorrhage of $15 \mathrm{~cm}^{3}$ or less have a better prognosis. ${ }^{3}$ A proportion of patients suffer massive intraventricular haemorrhage, usually from a ruptured anterior communicating artery aneurysm or intracerebral bleeding from a middle cerebral artery aneurysm. ${ }^{4}$ The exact cause of death when blood is confined to the subarachnoid space is unclear. Death may be associated with a rapid rise in intracranial pressure due to a sudden rush of blood at arterial pressure into the subarachnoid space.

Vasospasm of major cerebral arteries is seen mainly between two and seven days of subarachnoid haemorrhage or later. It is detectable by angiography ${ }^{5}$ and may significantly reduce blood flow, as measured by Doppler techniques. ${ }^{6}$ Vasospasm may complicate surgical clipping of an aneurysm and may result in extensive cerebral infarction; it is a major cause of death or disability in $13.5 \%$ of patients in hospital trials for the treatment of subarachnoid haemorrhage. ${ }^{7}$ However, arterial vasospasm and infarction contribute to only about $5 \%$ of all deaths during the first 30 days after subarachnoid haemorrhage.

Hydrocephalus may be seen in the acute stages of subarachnoid haemorrhage or may occur as a late complication. A small proportion of patients show ventricular dilatation on their first CT scan ${ }^{8}$; hydrocephalus at this stage may be caused by impedance of CSF flow through the blood filled subarachnoid space. At a later stage, often years after the subarachnoid haemorrhage, patients may develop intermittently raised pressure (normal pressure) hydrocephalus with the insidious onset of dementia, ataxia and incontinence. ${ }^{9}$ Hydrocephalus at this stage is probably caused by organisation of the subarachnoid blood clot, resulting in fibrous adherence of the arachnoid mater to the pia and to the surface of the brain, possibly impeding the flow of CSF through the subarachnoid space. There is little evidence that the arachnoid granulations become occluded ${ }^{1}$; although red blood cells may enter channels within arachnoid granulations following a subarachnoid haemorrhage, ${ }^{10}$ the granulations do not seem to become occluded by fibrous scar tissue.

Pathology of subarachnoid haemorrhage

Rupture of a saccular cerebral artery aneurysm accounts for 70-90\% of all subarachnoid haemorrhages in subjects over 30 years of age. ${ }^{3}$ Other causes, such as ruptured arteriovenous malformations, intracranial artery dissections, mycotic aneurysms, trauma, bleeding disorders, and a cervical origin for haemorrhage should be considered in the $15-20 \%$ of patients in whom no aneurysm is discovered on initial angiogram. ${ }^{111}$ Although saccular aneurysms are 


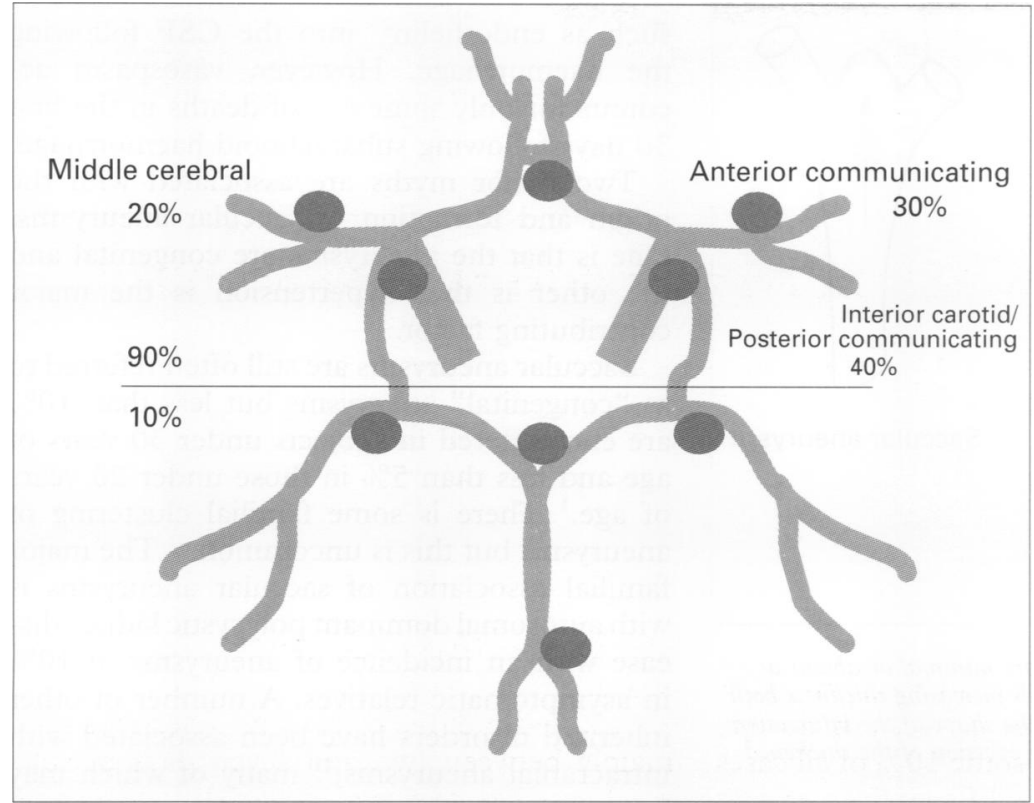

Figure 1 Distribution of saccular aneurysms on the circle of Willis.

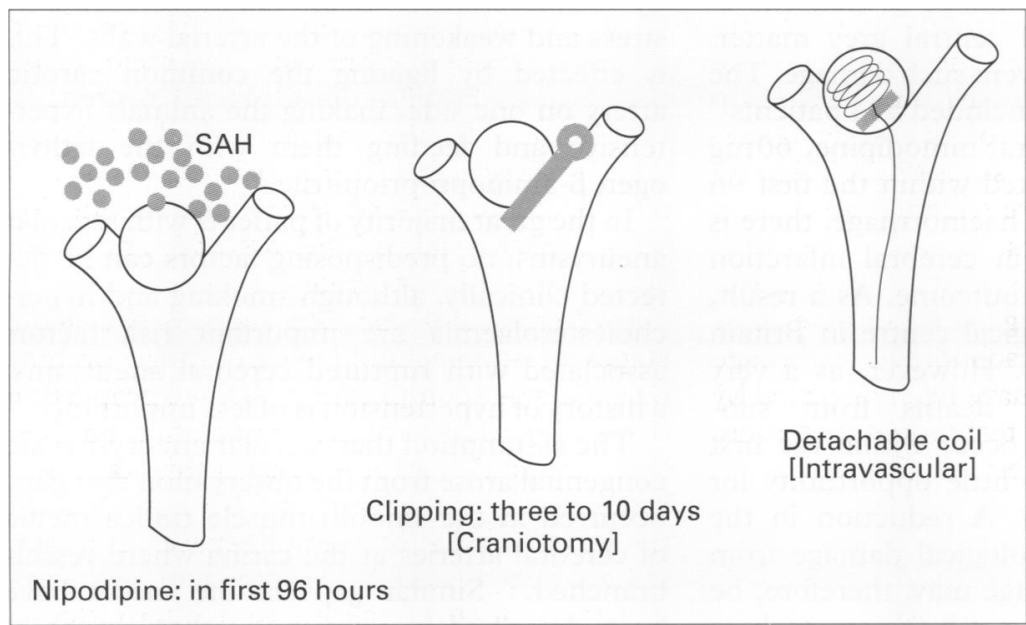

Figure 2 Summary of the techniques for the treatment of saccular aneurysms and their complications. SAH=subarachnoid haemorrhage.

found in an estimated $5 \%$ of the population, rupture occurs in only about $0.01 \%$ of the population per year. ${ }^{3}$ Ruptures occur most frequently on the intracranial portions of the internal carotid arteries ( $40 \%$ ) usually at the origins of the posterior communicating arteries, on the anterior communicating artery $(30 \%)$, and on proximal branches of the middle cerebral arteries $(20 \%)$ (fig 1 ). The remainder arise from the posterior cerebral, basilar and vertebral arteries and their branches. ${ }^{1213}$

At necropsy, subarachnoid haemorrhage is seen as a thin coating of blood in the subarachnoid space over the surface of the brain and as a rather thicker layer of blood around the ruptured aneurysm. The basal CSF cisterns are often filled with blood which can be visualised by CT scan. If bleeding is focal, it may indicate the location of an aneurysm but false localisation may occur if haemorrhage is intraventricular and has leaked through the foramina of Luschka into the pontine cisterns around the basilar artery. Preliminary removal of fresh blood clot from around the circle of
Willis may reveal the aneurysm or may facilitate dissection of the aneurysm in the fixed brain. If the blood clot around the aneurysm is allowed to harden by fixation, the aneurysm may be difficult to locate or it may be damaged during the removal of the solidified blood clot.

Multiple aneurysms occur in $25 \%$ of cases. ${ }^{1}$ In the series of 109 ruptured aneurysms reported by Inagawa and Hirano, ${ }^{4} 17 \%$ were less than $4 \mathrm{~mm}$ in diameter, $40 \%$ were $5-9 \mathrm{~mm}$, and $38 \%$ were $10 \mathrm{~mm}$ or larger in diameter. Giant aneurysms, greater than $25 \mathrm{~mm}$ in diameter, also occur. One third of aneurysms bleed and others present as mass lesions, either compressing the brain or resulting in temporal lobe epilepsy and in other focal neurological signs. Often with a thick fibrous and calcified wall, giant aneurysms may be filled with partly organised lamellated thrombus and appear as dense, rounded bodies on CT scan. Angiography may show a lumen very much smaller than the total capacity of the aneurysm.

If the results of angiography are available, they usually greatly facilitate the location of a saccular aneurysm at necropsy. Whether the location of the aneurysm is known or not, there are two major choices for examination of the brain. The circle of Willis and its major branches can be dissected free from the brain and the location of the aneurysm firmly identified on the exposed vessels. However, this procedure interferes with the relations among the aneurysm, the brain and the haemorrhage. Furthermore, if the ruptured aneurysm is very small, it may not be detectable on the dissected circle of Willis. Alternatively, therefore, it is often best to section the fixed brain coronally in $1 \mathrm{~cm}$ slices, find the point of haemorrhage to locate the aneurysm and then examine it macroscopically and histologically in relation to the artery and adjacent brain. In this way, the full effects of the ruptured aneurysm on the brain can be recorded more accurately.

In many cases of ruptured saccular aneurysm there is significant intraventricular or intracerebral haemorrhage. In a necropsy study of 133 patients with ruptured saccular aneurysms, ${ }^{4}$ intraventricular haemorrhage was observed in $40 \%$, half of which were caused by rupture of an anterior communicating artery aneurysm either through the inferior medial portion of the frontal lobe or through the corpus callosum. Intracerebral haemorrhage was seen in $39 \%$ of cases, caused by middle cerebral artery aneurysms in half of these.

\section{Treatment}

Therapy in subarachnoid haemorrhage is directed mainly towards prevention of neuronal damage in the brain and rebleeding from the aneurysm (fig 2). Nimodipine, a calcium channel blocker, is administered within the first 96 hours in an attempt to prevent neuronal damage. Red blood cells entering the subarachnoid space following rupture of an aneurysm are prevented from passing into perivascular spaces around arteries in the brain by the pia mater. ${ }^{14}$ It is probable, however, that soluble factors from the blood penetrate the 


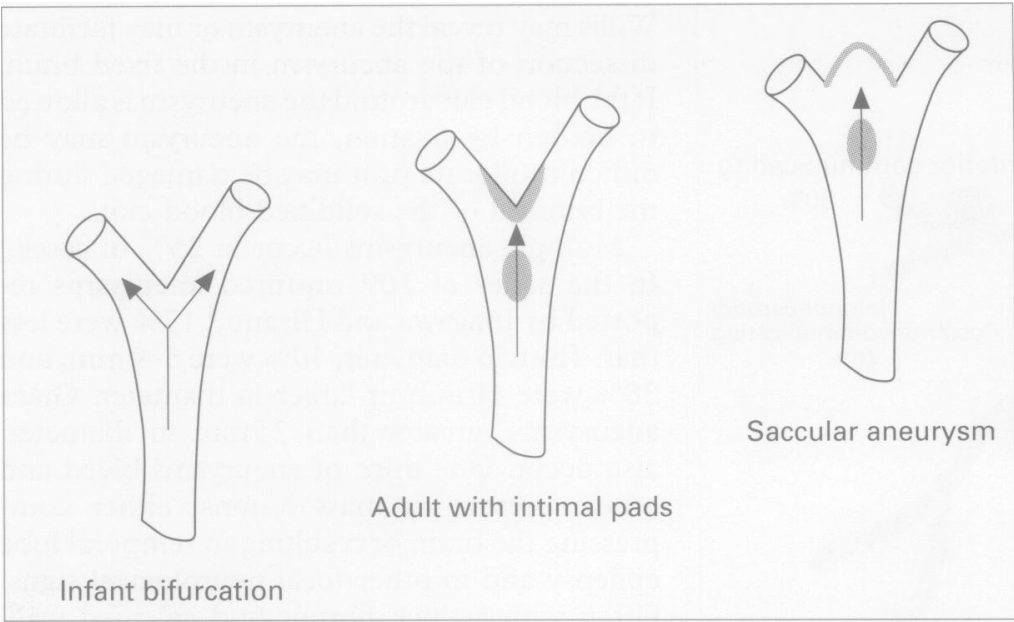

Figure 3 Development of saccular aneurysms. Intimal pads are minimal or absent at bifurcations of cerebral arteries in infants, but they develop with increasing thickness both proximal to and at vessel bifurcations in adults, thus altering the shape of the bifurcation and its haemodynamics. Saccular aneurysms could develop by eversion of the thickened area of the intimal pad. such as endothelin ${ }^{20}$ into the CSF following the haemorrhage. However, vasospasm accounts for only some $6 \%$ of deaths in the first 30 days following subarachnoid haemorrhage.

Two major myths are associated with the origin and formation of saccular aneurysms. One is that the aneurysms are congenital and the other is that hypertension is the major contributing factor.

Saccular aneurysms are still often referred to as "congenital" aneurysms but less than $10 \%$ are encountered in subjects under 30 years of age and less than $5 \%$ in those under 20 years of age. ${ }^{1}$ There is some familial clustering of aneurysms but this is uncommon. ${ }^{21}$ The major familial association of saccular aneurysms is with autosomal dominant polycystic kidney disease with an incidence of aneurysms of $10 \%$ in asymptomatic relatives. A number of other inherited disorders have been associated with intracranial aneurysms, ${ }^{21}$ many of which may be associated with structural abnormalities of the arterial walls. Aneurysms on cerebral arteries can be induced in experimental animals by a combination of altered haemodynamic stress and weakening of the arterial walls. This is effected by ligating the common carotid artery on one side, making the animals hypertensive and feeding them with the lathyrogen $\beta$-aminoproprionitrile. ${ }^{22}$

In the great majority of patients with saccular aneurysms, no predisposing factors can be detected clinically, although smoking and hypercholesterolaemia are important risk factors associated with ruptured cerebral aneurysms; a history of hypertension is of less importance. ${ }^{16}$

The assumption that saccular aneurysms are congenital arose from the observation that gaps occurred in the smooth muscle tunica media of cerebral arteries at the carina where vessels branched. ${ }^{23}$ Similar gaps in the media have been described in arteries in other organs of the body. ${ }^{24}$ It was thought, however, that the gaps presented points of weakness which led directly to the formation of saccular aneurysms. In a survey of cerebral artery bifurcations in subjects between one and 80 years of age, gaps in the media at the distal carina of bifurcations were found in $60 \%$ of cases, and the gaps enlarged with increasing age. ${ }^{24}$ The same survey showed that intimal pads, with duplication and fragmentation of the internal elastic lamina, formed at the bifurcations and proximal to bifurcations in arteries of older patients. Such pads were seen at $22 \%$ of bifurcations by 10 years of age and at $60 \%$ of bifurcations over the age of 30 years. ${ }^{24}$ The increase in luminal pads proximal to and at bifurcations of cerebral arteries with age has two major effects. Firstly, the pads induce focal loss of elasticity of the arterial wall and, secondly, the shape of the carina of the artery changes to become more rounded (fig 3). Theoretically, the changes at the bifurcation could alter the direction of haemodynamic forces on the carina so that haemodynamic stress may be more directed at the rounded apex of the carina (fig 3 ). In this way, the rounded carina is gradually pushed out to form an aneurysm at the point of vessel branching (fig 3). Histological examination chnoid haemorrhage. no doubt that vasospasm occurs ${ }^{5}$ and that it may result in decreased blood flow ${ }^{6}$ and infarction. The cause of vasospasm is not entirely clear and it may be due to the release of factors 
shows how the smooth muscle coat and the elastic lamina of the artery stop abruptly at the neck of the saccular aneurysm. ${ }^{125}$ The wall of the aneurysm is thus formed entirely from fibrous tissue and endothelium with or without thrombus coating its luminal surface. As aneurysms also occur at points at which vessels curve, similar forces may be in action in these regions.

The question still remains as to why aneurysms only form in $5 \%$ of the population. Development of aneurysms may depend on a combination of haemodynamic forces and the configuration of the vessel bifurcations. There is considerable individual variation in the pattern of vessels around the circle of Willis, ${ }^{26}$ particularly in the calibre of vessels such as the posterior communicating and anterior cerebral arteries; this could affect the pattern of stress on points of branching. Furthermore, there is a high incidence of aneurysms on arteries feeding arteriovenous malformations ${ }^{1}$ and aneurysms are associated with vascular abnormalities such as Moya Moya disease, in which there is aggressive occlusion of the internal carotid artery and its branches with the formation of "netlike" vessels. ${ }^{27}$ These observations suggest that blood flow patterns may play a role in the formation of aneurysms, possibly by inducing intimal pads within the arteries, altering the shape of vessel branches, and thus increasing susceptibility to the development of aneurysms.

The last important question is why do aneurysms rupture? Thinning of the fibrous wall of the aneurysm may occur and rupture frequently occurs at the apex of the aneurysm. Although it was originally suggested that hypertension is a major factor involved in the formation of aneurysms, it appears to be less important than risk factors such as smoking and increased serum cholesterol. ${ }^{1628}$ Subarachnoid haemorrhage tends to occur in ambulatory patients and following physical exertion; a sudden rise in blood pressure may therefore play a part in rupture of aneurysms. It has also been suggested that changing weather conditions precede aneurysm rupture in men but not in women. ${ }^{29}$ Men show a single large peak of aneurysm rupture in the late autumn, whereas in women the annual peak occurs in late Spring.

In conclusion, pathologists have an important role to play in increasing our understanding of the multiple factors in the environment, in the structure and branching patterns of the circle of Willis, and in the genetic make-up of subjects that result in the formation and rupture of saccular aneurysms. Such an understanding will allow preventative measures to be adopted to help reduce the very high mortality and morbidity associated with saccular aneurysms and subarachnoid haemorrhage.
1 Weller RO. Spontaneous intracranial haemorrhage. In: Adams JH, Duchen LW, eds. Greenfield's Neuropathology. 5th edn. London: Edward Arnold, 1992:269-301.

2 Broderick JP, Brott TG, Duldner JE, Tomsick T, Leach A. Initial and recurrent bleeding are the major causes of death following subarachnoid haemorrhage. Stroke 1994; 25:1342-7.

3 Sacco RL, Wolf PA, Kannel WB, McNamara PM. Survival and recurrence following stroke. The Framingham Study. Stroke 1982;13:290-5.

4 Inagawa $T$, Hirano A. Ruptured intracranial aneurysms: an autopsy study of 133 patients. Surg Neurol 1990;33: $117-23$.

5 Niizuma H, Kwak R, Otake K, Suzuki J. Angiographic study of cerebral vasospasm following the rupture of intracranial aneurysms II; relation between the site of aneurysm and aneurysms II; relation between the site of aneurysm and the occurrence of vasospasm. Surg Neurol 1979;11:263-7.

6 Sloan MA, Burch CM, Wozniak MA, Rothman MI, Rigamonti D, Permutt T, et al. Transcranial Doppler detection of vertebrobasilar vasospasm following subarachnoid hemorrhage. Stroke 1994;25:2187-97.

7 Kassell NF, Torner JC, Clarke Haley E, Jane JA, Adams HP, Kongable GL, et al. The international co-operative study on the timing of aneurysm surgery. Part 1: Overall management results. $\mathcal{F}$ Neurosurg 1990;73:18-36.

8 Heros RC. Acute hydrocephalus after subarachnoid hemorrhage. Stroke 1989;20:715-17.

9 Pickard JD Adult communicating hydrocephalus. $B r f H o s p$ Med 1982;27:35-44.

10 Upton ML, Weller RO. The morphology of cerebrospinal fluid drainage pathways in human arachnoid granulations. f Neurosurg 1985;63:867-75.

11 Rinkel GJE, Gijn JV, Wijdicks EFM. Subarachnoid haemorrhage without detectable aneurysm. A review of the causes. Stroke 1993;24:1403-9.

12 Graham DI. Vascular disease and hypoxic brain damage. In: Weller RO, ed. Nervous system, muscle and eyes: systemic pathology, 3rd edn. Vol 4. Edinburgh: Churchill Livingstone, 1990:89-124.

13 Ujiie H, Sato K, Onda H, Oikawa A, Kagawa M, Takakura $\mathrm{K}$, Kobayashi N. Clinical analysis of incidentally discovered aneurysms. Stroke 1993;24:1850-6.

14 Hutchings $M$, Weller RO. Anatomical relationships of pia mater to cerebral blood vessels in man. $\mathcal{F}$ Neurosurg 1986; 65:316-25.

15 Pickard JD, Murray GD, Illingworth R, Shaw MDM, Teasdale GM, Foy PM, et al. Effect of oral nimodipine on cerebral infarction and outcome after subarachnoid haemorrhage. British aneurysm nimodipine trial. $B M \mathcal{F}$ 1989;298:636-42.

16 Adamson J, Humphries SE, Ostergaard JR, Volby B, Richards P, Powell JT. Are cerebral aneurysms atherosclerotic? Stroke 1994;25:963-6.

17 Drake CG, Peerless SJ, Ferguson GG. Hunterian proximal arterial occlusion for giant aneurysms of the carotid circulation. $\mathcal{F}$ Neurosurg 1994;81:656-65.

18 Guglielmi G, Fernando V, Dion J, Duckwiler G. Electrothrombosis of saccular aneurysms via endovascular approach. Part 2: Preliminary clinical experience. $\mathcal{F}$ Neurosurg 1991;14:75-8.

19 van Halbach V, Higashida RT, Dowd CF, Barnwell SL, Fraser KW, Smith TP, et al. The efficacy of endosaccular aneurysm occlusion in alleviating neurological deficits produced by mass effect. $\mathcal{F}$ Neurosurg 1994;80:659-66.

20 Seifert V, Löffler B-M, Zimmerman M, Roux S, Stolke D. Endothelin concentrations in patients with aneurysmal subarachnoid hemorrhage. Correlation with cerebral vasosubarachnoid hemorrhage. Correlation wicits and volume spasm, delayed ischemic neurological deficits

21 Schievink WI, Schaid DJ, Rogers HM, Piepgras DG, Michels VV. On the inheritance of intracranial aneurysms. Stroke 1994;25:2028-37.

22 Hazama F, Hashimoto N. An animal model of cerebral aneurysms. Neuropathol App Neurobiol 1987;13:77-90.

23 Forbus WD. On the origin of miliary aneurysms of the superficial cerebral arteries. Bulletin of the fohns Hopkins Hospital 1930;47:239-84.

24 Sheffield EA, Weller RO. Age changes at cerebral artery bifurcations and the pathogenesis of berry aneursyms. $\mathcal{F}$ Neurol Sci 1980;46:341-52.

25 Weller RO. Colour atlas of neuropathology. Oxford: Harvey Miller and Oxford University Press, 1984:31.

26 Mackenzie JM. The anatomy of aneurysm-bearing circles of Willis. Clin Neuropathol 1991;10:187-9.

27 Massoud TF, Guglielmi G, Vinuela F, Duckwitler GR Saccular aneurysms in Moyamoya disease: endovascular treatment using electrically detachable coils. Surg Neurol 1994;41:462-7.

28 McCormick WF, Schmalstein EJ. The relationship of arteria hypertension to intracranial aneurysms. Arch Neurol 1977 34:285-7.

29 Chyatte D, Chen TL, Bronstein K, Brass LM. Seasonal fluctuation in the incidence of intracranial aneurysm rupture and its relationship to changing climatic conditions. f Neurosurg 1994;81:525-30. 